\title{
Frontières
}

\section{HANUS, Michel, La résilience à quel prix ? Survivre et rebondir, Paris, Maloine, 2001, 231 p.}

\section{Denise Badeau}

Volume 15, numéro 2, printemps 2003

URI : https://id.erudit.org/iderudit/1073832ar

DOI : https://doi.org/10.7202/1073832ar

Aller au sommaire du numéro

\section{Éditeur(s)}

Université du Québec à Montréal

\section{ISSN}

1180-3479 (imprimé)

1916-0976 (numérique)

Découvrir la revue

Citer ce compte rendu

Badeau, D. (2003). Compte rendu de [HANUS, Michel, La résilience à quel prix ? Survivre et rebondir, Paris, Maloine, 2001, 231 p.] Frontières, 15(2), 87-88.

https://doi.org/10.7202/1073832ar d'utilisation que vous pouvez consulter en ligne.

https://apropos.erudit.org/fr/usagers/politique-dutilisation/ 
réalité ancienne. L'auteur, bien connu pour ses ouvrages sur le deuil, annonce d'entrée de jeu, que ce travail sur la résilience ne vise pas à être scientifique. "Il va s'efforcer d'être une œuvre de réflexion qui s'autorisera plusieurs hypothèses après avoir soulevé de nombreuses questions, sans nécessairement y répondre " (p. 10). De fait, le deuil est une porte d'entrée intéressante, invitante dans l'édifice de la résilience. "[...] comme le deuil, la résilience est un processus qui s'initie durant la prime enfance, se développe et se manifeste durant toute la vie (et au-delà de la vie ?) » (p. 12). Elle peut apparaître comme " un avatar particulier de l'aptitude au deuil » (p. 12).

Pour alimenter sa réflexion, Michel Hanus utilisera plusieurs ouvrages, surtout l'article de Michaël Rutter de 1985: Resilience in the Face of Adversity, les ouvrages de Boris Cyrulnic: Un merveilleux malheur (1999), qui le laisse à la fois "ébloui et insatisfait " tandis que Les vilains petits canards (2001) lui permet d'avancer dans la compréhension de la nature et des origines de la résilience; et enfin, I'ouvrage de Pierre Rentchnick, André Haynal et Pierre de Senarclens: Les Orphelins mènent-ils le monde? (1978). II affirme avoir conservé cet intérêt à l'état latent pour ce qu'il n'appelait pas encore "résilience", mais qu'il voyait comme "une capacité psychique à rebondir sur les épreuves ».

\section{VERS UNE DÉFINITION}

Le terme résilience semble venir de la physiopathologie, domaine dans lequel elle signifie habituellement un mode de résistance à certains agents pathogènes; la résistance serait l'effet du processus de résilience mais ne s'y réduit pas. Elle serait un ensemble de processus dynamiques interactifs évoluant dans le temps et selon le jeu des circonstances. Pour Michaël Rutter :

La résilience se caractérise par un type d'activité qui met en place dans l'esprit un but et une sorte de stratégie pour réaliser

l'objectif choisi, les deux paraissant comporter plusieurs élé-

ments connectés: une estime de soi et une confiance en soi suffisantes, la croyance en son efficacité personnelle et la disposition d'un répertoire de solutions.

Elle est nettement influencée par deux facteurs de protection: des relations affectives

sécurisantes et stables et des expériences de succès et de réussites (p. 24).

Michel Hanus voit là une tentative d'explication plutôt qu'une définition. Après à ce travail sur l'article de Rutter, l'auteur retient que la résilience est avant tout une forme de résistance au stress, "le concept étant tout autant centré sur le processus opérant que sur ses résultats qui interfèrent rétroactivement avec lui pour le modifier quantitativement et qualitativement, dans ses mécanismes qui sont adaptatifs " (p. 45). La résilience est aussi un élément essentiel du développement; en ce sens, chacun porte en lui des capacités de résilience. Pour se constituer, encore faut-il arriver à surmonter des épreuves avec succès augmentant ainsi l'estime de soi et la confiance dans sa propre efficacité. La résilience protège et dynamise. Il est difficile de parler de résilience sans l'inscrire dans une temporalité déterminée, "hors de laquelle elle n'est encore que de l'ordre du possible, ou n'est déjà plus qu'un souvenir ". La résilience fonctionnerait comme symptôme exprimant la " difficulté qui l'a fait naître, sa permanence et, dans le temps, conjointement, le traitement qu'on lui a fait subir pour lui faire face ", et comme formation réactionnelle: " cette force qui se manifeste à l'extérieur tirant ses forces [...] de celles de la souffrance méconnue ». Vue à la fois comme œuvre de révolte contre une difficulté particulièrement lourde ressentie par l'enfant, comme un message, une réponse adressée à un ou des interlocuteurs, la résilience semble souvent comporter une dimension de création (p. 45-47).

Boris Cyrulnic, quant à lui, rapporte la définition de $S$. Vanistendael : "la capacité à réussir, à vivre et à se développer positivement, de manière socialement acceptable, en dépit du stress ou d'une adversité qui comportent normalement le risque grave d'une issue négative» (1996), « bref s'épanouir malgré le malheur et, en quelque sorte, grâce à lui en tant que révélateur de potentialités personnelles encore latentes jusqu'alors ». « [...] il se maintiendra entre ses deux positions survivre et rebondir - mais en privilégiant la première celle de la résistance »- le terme étant d'abord physique - "alors que les trois auteurs suisses s'étaient davantage tournés vers la dimension de la créativité » (p. 87).

Résistance, la résilience $n$ 'est pas que cela. " Un des intérêts de la résilience, de son étude et de sa compréhension est de laisser les pronostics ouverts pour tous ces enfants maltraités de quelque manière ». On reconnaît que certains facteurs aggravent les traumatismes mais notre appréciation extérieure des quantités et des qualités de la souffrance d'autrui demeurent approxi- mative et subjective. La résilience, nous l'apprécions en fonction du décalage que nous percevons entre le ou les traumatismes que la personne a subis et ses réactions actuelles reconnues particulièrement positives (p. 131-132). «le premier traumatisme oblige, pour des raisons de survie, à développer ces potentialités, le second est l'occasion de leur révélation » (p. 143).

D'autre part, Cyrulnic «essaie surtout de faire prendre conscience de l'existence et de l'importance de la résilience, qu'elle a une forme et qu'elle coûte un prix plutôt que de tenter d'en expliquer les origines, les fondements et les modes de fonctionnement ", ce qui est davantage le propos de Michel Hanus. "Tous les chercheurs et praticiens qui élaborent ce concept s'intéressent maintenant à la manière dont elle se construit. D'après les travaux en cours, la génétique aura son mot à dire mais les interactions précoces parleront beaucoup plus tandis que les institutions familiales et sociales tiendront l'essentiel du discours» (p. 87-88). Dans Les vilains petits canards, la résilience « apparaît résolument comme une capacité ou un ensemble de capacités, et un processus tout à fait général, universel peut-être, dans le développement: la réussite à surmonter les épreuves ". II y a ainsi comme une résilience ordinaire et une résilience exceptionnelle. Cyrulnic se place dans deux dimensions: celle du temps (la dynamique) et celle de l'interaction (p. 88).

Michel Hanus rebondit sur certaines idées susceptibles de venir éclairer ses interrogations:

Si la résilience a besoin d'un malheur, d'un traumatisme, en deux temps pour pouvoir s'exprimer, elle nécessite également une ou plusieurs rencontres significatives avec un adulte suffisamment attentif et bienveillant pour aider à restaurer en partie la confiance dans les autres et dans le monde, et indirectement en soi. L'histoire de la résilience est nécessairement traumatique réalisant une élaboration particulière des agressions graves. La résilience est également une œuvre de révolte, elle est le fruit de la colère, elle tire ses forces de l'agressivité que le traumatisme a fait naître ou vient réveiller. Enfin, la résilience, si elle s'inscrit dans la temporalité, est un authentique processus au long cours au sein de la vie psychique (p. 22).

La résilience est-elle une forme de sublimation? Ce qui incite à les rapprocher, selon Hanus, c'est: attribuables à son appellation récente, la résilience n'en est pas moins une 
en premier lieu, qu'elles témoignent l'une et l'autre d'une grande force, de la mobilisation de grandes forces. En second lieu, leur résultat paraît dépasser largement leurs prémisses. L'une et l'autre sont des processus de création. Enfin, cette œuvre nous touche de l'extérieur, elle nous parle; nous nous $y$ retrouvons en quelque sorte.

Plus encore, elle montre advenu ce que nous voudrions être. Mais, comme nous ne sommes les auteurs ni de l'une ni de I'autre, nous aurions tendance à en sous-estimer la peine, la gestation créatrice de la sublimation, les souffrances endurées au départ de la résilience (p. 162).

Capacité, aptitude, habileté particulière à certaines personnes, la résilience leur permet de réagir avec force à un moment précis en présence d'épreuves majeures qui sembleraient à première vue devoir plutôt les écraser ou les submerger. Elles se servent même de ces épreuves pour résister au stress, pour progresser, intégrer le malheur et en sortir plus fortes, plus aguerries. Pour comprendre la résilience dans sa nature profonde, comme dans ses origines, il paraît indispensable de considérer d'abord séparément chacun des deux facteurs, la personnalité d'abord puis les traumatismes sans oublier le contexte et l'entourage (p. 107-108).

La rêverie, un monde psychique imaginaire, le déni, l'intellectualisation, les moyens d'expression, I'humour, le dessin, le soutien extérieur, le soutien affectif sont autant de moyens de réagir activement à la situation traumatisante, de reprendre l'initiative, de faire quelque chose pour soi-même pour s'en sortir ou, au moins, si ce n'est pas possible dans l'immédiat, pour améliorer la situation. Cyrulnic voit la résilience comme un processus de résistance, de restauration, et encore plus que cela, un ressort ou un rebond. Hanus retient que "la résilience est une interaction dynamique à un moment déterminé entre un individu confronté à un événement exceptionnellement traumatisant, ce qui lui permet, au fil du temps, de mettre en action un processus de restauration dont nous pouvons, de l'extérieur, constater les effets, là encore à un moment déterminé et dans un contexte donné, processus qui semble permettre à l'intéressé de voir croître ses possibilités de développement personnel entraînant des effets positifs inattendus ". L'auteur souscrit à l'affirmation de B. Cyrulnic que la résilience a un prix et nous invite à n'apprécier les effets de la résilience, aussi brillants qu'ils puissent paraître, que resitués dans l'ensemble du fonctionnement et de la dynamique de cette personne.

"Présentée comme un symptôme positif, i.e. à la fois comme une expression et une porte de sortie du traumatisme, la résilience comporte alors un double envers: le prix qu'elle coûte, la dépense énorme d'énergie qu'elle demande, la fatigue qu'elle entraîne et peut-être un épuisement plus précoce... » (p. 195).

\section{DE LA RÉSILIENCE ET DU DEUIL}

D'emblée, la résilience et le deuil présentent des points communs: ils s'expriment dans le temps, ils sont les manifestations plus ou moins visibles d'un travail intérieur, ils sont provoqués par la perte, ils aboutissent, à plus ou moins long terme, à des résultats. "L'un et l'autre se situent dans une histoire avec son développement dynamique, conditionné en partie par les antécédents mais toujours ouverte aux aléas de l'existence qui ne sont pas toujours que négatifs. La résilience et le deuil vont tirer bénéfice de tout support relationnel affectif dont les personnes concernées sont susceptibles de pouvoir bénéficier» (p. 51). La partie non terminée d'un deuil risque de refaire surface lors de la prochaine épreuve ou du prochain deuil alors que la résilience apparaît comme une œuvre de déni, déni de sa signification psychique, déni de la souffrance. Alors que la régression est un élément essentiel du deuil, la résilience apparaît au contraire comme " un grand bond en avant ". À l'opposé du deuil, elle n'apparaît pas comme une solution durable. "Elle coûte trop cher; elle est trop défensive, elle n'est pas naturelle. Plus elle semble perdurer, plus elle brûle d'énergie, plus elle épuise. Lorsqu'elle arrive à se maintenir, c'est aux dépens d'autres secteurs de la personnalité » (p. 61).

Selon l'auteur, la résilience et le deuil entretiennent des relations mitigées. Ils sont l'une et l'autre "comme des processus dont les origines et les modalités de fonctionnement retiendront davantage l'attention et l'intérêt que leurs effets, leurs résultats » (p. 70). Tout comme le deuil, la résilience est activité psychique et activité dans le monde, dans le corps social sous l'égide de la solidarité. Le deuil se vit dans la souffrance, la douleur morale, la résilience s'y enracine; I'un et l'autre s'efforcent de la dépasser. Le deuil implique un mouvement de régression dynamique, la résilience le bloque. Les deux, pour se réaliser, ont besoin d'une recon- naissance suffisante de la situation et objective et psychique qui va permettre d'entamer le travail intérieur qui, dans un cas, va du refus initial à I'acceptation progressive et jamais achevée et dans l'autre atteint son apogée. "Le résilient a non seulement fait face à l'épreuve, il est passé au-dessus. » Dans la résilience, «le parent imaginaire auquel l'enfant rêve n'est pas mort; c'est son parent actuel qu'il idéalise, ou plutôt qu'il ne peut cesser d'idéaliser... L'enfant résilient reconstruit son parent dans ses rêveries qui ont un rôle essentiel pour son équilibre plus encore que pour tout enfant qui en a déjà le plus grand besoin " (p. 191-192).

La résilience serait un processus analogue à celui du "deuil narcissique » à partir de la constitution de I' «aptitude au deuil». Cette aptitude au deuil regroupe cinq éléments que nous trouvons également dans la résilience: « un monde interne constitué, une indépendance psychique relative, des cognitions suffisantes (connaissance, reconnaissance des situations stressantes), le vécu émotionnel qui a besoin de la rencontre d'un adulte bienveillant lorsque cet apport affectif fait défaut au sein de la famille et une sécurité élémentaire auquel l'enfant résilient peut lui-même participer ". Un deuil révèle l'état du précédent: si le deuil précédent a pu être correctement intégré, un nouveau deuil se fera correctement... La personne résiliente peut devenir plus forte d'épreuve surmontée en épreuve surmontée mais jusqu'à quelle limite? Ou bien une nouvelle épreuve aura-t-elle raison de sa résilience... » (p. 193-195).

\section{QUI SONT LES ENFANTS \\ RÉSILIENTS ?}

Ont-ils reçu un bagage génétique, des forces et des qualités éventuellement peu perceptibles de l'extérieur, qui se manifesteront ultérieurement dans des moments difficiles, la rencontre avec le traumatisme servant de révélateur? «L'avis est unanime sur l'importance de la famille dans la formation des bases de la résilience puisque c'est en elle que s'initient et se développent la confiance de base, l'estime de soi, le sentiment d'identité et celui de ses propres qualités opérationnelles qui sont les conditions indispensables, sinon les éléments constitutifs de la résilience » (p.140).

Le résilient fait mieux que de survivre au traumatisme, il le transcende, l'intègre, le surpasse. II transforme "ses forces destructrices en élans constructeurs". Les énergies qu'il emploie à l'affronter semblent se convertir en expressions de poten-

tiels jusque-là latents. La résilience est intime, secrète, en bonne partie, inconsciente dans ses "élaborations processuelles "... Elle est construction, création, œuvre narcissique qui nous émeut. "Elle incarne la présence du Moi idéal, elle le substantifie, elle le fait advenir. Tout simplement, la résilience fait miroiter la possibilité d'une toutepuissance retrouvée, au moins chez un autre à défaut d'être en nousmêmes" (p. 145).

\section{DES HYPOTHÈSES}

Les hypothèses formulées par l'auteur portent sur la constitution et la mise en place de la résilience, sur sa face sombre, sur sa caractéristique en tant qu'œure de révolte, fruit de la colère, sur la résilience comme processus psychique, sur l'importance des parents, de la famille sur le plan génétique et éducatif, sur la rencontre, la chance d'une relation satisfaisante.

$$
\text { *** }
$$

Porteuse d'espérance, "la résilience a manifestement deux sens principaux [...] un sens large et général, elle qualifie tous les aspects dynamiques du développement psychologique à la fois dans ses processus, ses capacités, ses modes opératoires, ses expressions concrètes et en ce sens, elle est présente en chacun " ou résilience habituelle; un sens plus précis: survivre et rebondir, "faire preuve de capacités hors du commun de mettre en évidence un écart inhabituel entre la profondeur du traumatisme et une réussite inattendue dans de telles circonstances " ou résilience inhabituelle. Hanus nous invite à les soutenir l'une et l'autre en faisant sentir à la personne ou en lui disant avec des mots discrets et adéquats que l'on connaît et que I'on reconnaît le caractère traumatique de sa situation, de sa souffrance. "Favoriser la résilience, I'accompagner, la soutenir, l'aider, c'est lutter contre les facteurs de risque et optimiser les facteurs de protection. " "L'enfant résilient, tout résilient qu'il soit est aussi un être blessé. La résilience dont il fait preuve lui permet à la fois de survivre et aussi d'aller plus loin (rebondir) mais elle ne supprime pas la blessure » (p. 214).

Cet ouvrage de Michel Hanus nous a permis de nourrir notre réflexion sur la nature de la résilience, sur ses éléments constitutifs, ses effets. Mais, avec lui, nous formulons le souhait que la recherche tant fondamentale qu'expérimentale se poursuive sur le sujet afin de mieux cerner la réalité présente et future des enfants résilients.

Denise Badeau 